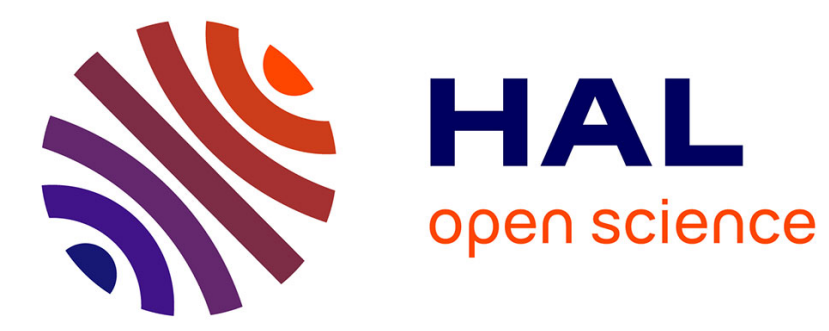

\title{
Uplink Transmission Probability Functions for LoRa-Based Direct-to-Satellite IoT: A Case Study
}

Kai Vogelgesang, Juan Andrés A Fraire, Holger Hermanns

\section{To cite this version:}

Kai Vogelgesang, Juan Andrés A Fraire, Holger Hermanns. Uplink Transmission Probability Functions for LoRa-Based Direct-to-Satellite IoT: A Case Study. GLOBECOM 2021 - IEEE Global Communications Conference, Dec 2021, Madrid, Spain. pp.1-6. hal-03494140

\author{
HAL Id: hal-03494140 \\ https://hal.science/hal-03494140
}

Submitted on 18 Dec 2021

HAL is a multi-disciplinary open access archive for the deposit and dissemination of scientific research documents, whether they are published or not. The documents may come from teaching and research institutions in France or abroad, or from public or private research centers.
L'archive ouverte pluridisciplinaire HAL, est destinée au dépôt et à la diffusion de documents scientifiques de niveau recherche, publiés ou non, émanant des établissements d'enseignement et de recherche français ou étrangers, des laboratoires publics ou privés. 


\title{
Uplink Transmission Probability Functions for LoRa-Based Direct-to-Satellite IoT: A Case Study
}

\author{
Kai Vogelgesang*, Juan A. Fraire*†, Holger Hermanns* \\ *Saarland University, Saarland Informatics Campus, Saarbrücken, Germany \\ ${ }^{\dagger}$ Univ Lyon, Inria, INSA Lyon, CITI, F-69621 Villeurbanne, France
}

\begin{abstract}
Direct-to-Satellite IoT allows devices on the Earth surface to directly reach Low-Earth Orbit (LEO) satellites passing over them. Although an appealing approach towards a truly global IoT vision, scalability issues as well as highly dynamic topologies ask for dedicated protocol adaptations supported by novel models. This paper contributes to this research by introducing estimators and a transmission probability function to dynamically control the contending set of devices on a framed slotted Aloha model compatible with the LoRaWAN specification. In particular, we discuss techniques that account for particularities in the dynamics of sparse DtS-IoT constellations. Simulation analyses of a realistic case study show that $>86 \%$ of the theoretical throughput is achievable in practice.

Index Terms-MAC, Direct-to-Satellite IoT, LoRaWAN.
\end{abstract}

\section{INTRODUCTION}

The internet of things (IoT) is just around the corner. Emerging standards such as $5 \mathrm{G}$ are designed to accommodate large numbers of machine-to-machine (M2M) communications [1] leveraging novel Low Power Wide Area (LPWA) technologies such as LoRaWAN, LR-FHSS and NB-IoT [2, 3, 4, 5]. Applications ranging from agriculture to smart grid, environmental monitoring, emergency management, among others can be unblocked by enabling the transmission of small amounts of information from long distances (100s km, at $<50 \mathrm{Kbps}$ ) [6]. By 2023, it is expected that more than 14 billion connections $-50 \%$ of all network connections- will involve massive, cheap and power-efficient IoT devices [7]. A global IoT service will thus be crucial to uphold the IoT trend. In this context, constellations of Low-Earth Orbit (LEO) satellites are central to support this ambitious goal [8].

The so-called Direct-to-Satellite IoT (DtS-IoT) paradigm constitutes the holy grail of satellite-based IoT [9]. The core idea is to succeed in connecting resource-constrained devices on ground directly to LEO satellites without relying on intermediate gateways [10]. DtS-IoT is particularly attractive in regions where deploying infrastructure is difficult, economically uninteresting (i.e., about half of the inhabited area of the planet [11]), or impossible due to disaster conditions. Recent in-orbit demonstration of the LoRaWAN-based LacunaSat-1 nano-satellite evidenced the practical feasibility and commercial attractiveness of the DtS-IoT concept [12]. Furthermore, recent work showed that DtS-IoT services can already be provided with just a dozen of satellites acting as opportunistic data mules [9] (a.k.a. sparse constellations, in contrast to resource-hungry mega-constellations).
The problem of DtS-IoT is that satellites orbiting in LEO move at more than $25,000 \mathrm{~km} / \mathrm{h}$, and so does their area coverage on the Earth surface. Furthermore, at altitudes in the order of $500 \mathrm{~km}$, a LEO satellite completes a pass over a serviced region in less than 10 minutes. Besides the challenge of coordinating the service to potentially thousands of IoT devices at the sight of the passing-by LEO satellite, the overall network dynamics of DtS-IoT are far from the use cases that are motivated by traditional terrestrial IoT protocols.

In this work, we tackle the medium access control challenges of sparse DtS-IoT. First, we propose a framed slotted Aloha [13] model that can be materialized with standard beacon-based LoRaWAN devices and gateways [14]. Secondly, we deal with the scalability aspect by providing an estimator of the successful extraction rate for a given amount of contending nodes. Based on this, we derive a Transmission Probability Function $(T P F)$ that, by beaconing the resulting value, enables an accurate control of the cardinality of the contending set during the frame, Thirdly, we uncover a non trivial problem in frame-based sparse DtS-IoT. Devices might receive the beacon but the fast-paced LEO satellite dynamics could leave them out-of-sight by the time an uplink transmission is scheduled. Since sparse constellations might not provide an immediate new satellite over the horizon, we propose two practical solutions to mitigate this issue: $i$ ) throttling the estimator to compensate, and ii) empower perceptive devices to account for trajectory information. Finally, we present a realistic DtS-IoT scenario with thousands of devices to evaluate and assess the proposed approaches.

The remainder of this paper is organized as follows: Section II introduces the framed slotted Aloha DtS-IoT model. The $T P F$ function, estimators and waste transmission mitigation schemes are introduced in Section III. Section IV presents a realistic case study where the methods are evaluated by means of simulations. Conclusions are drawn in Section V.

\section{DTS-IOT MODEL}

\section{A. Framed Slotted Aloha DtS-IoT}

Our DtS-IoT model, illustrated in Fig. 1, assumes that the communication between the satellite and the IoT devices on ground is organized in frames. The IoT gateway payload on the satellite transmits a periodic beacon to mark the beginning of a frame. A device that decodes the beacon is assumed to have the satellite in line of sight while enjoying good enough channel conditions to reach the gateway back. Thus, an uplink 

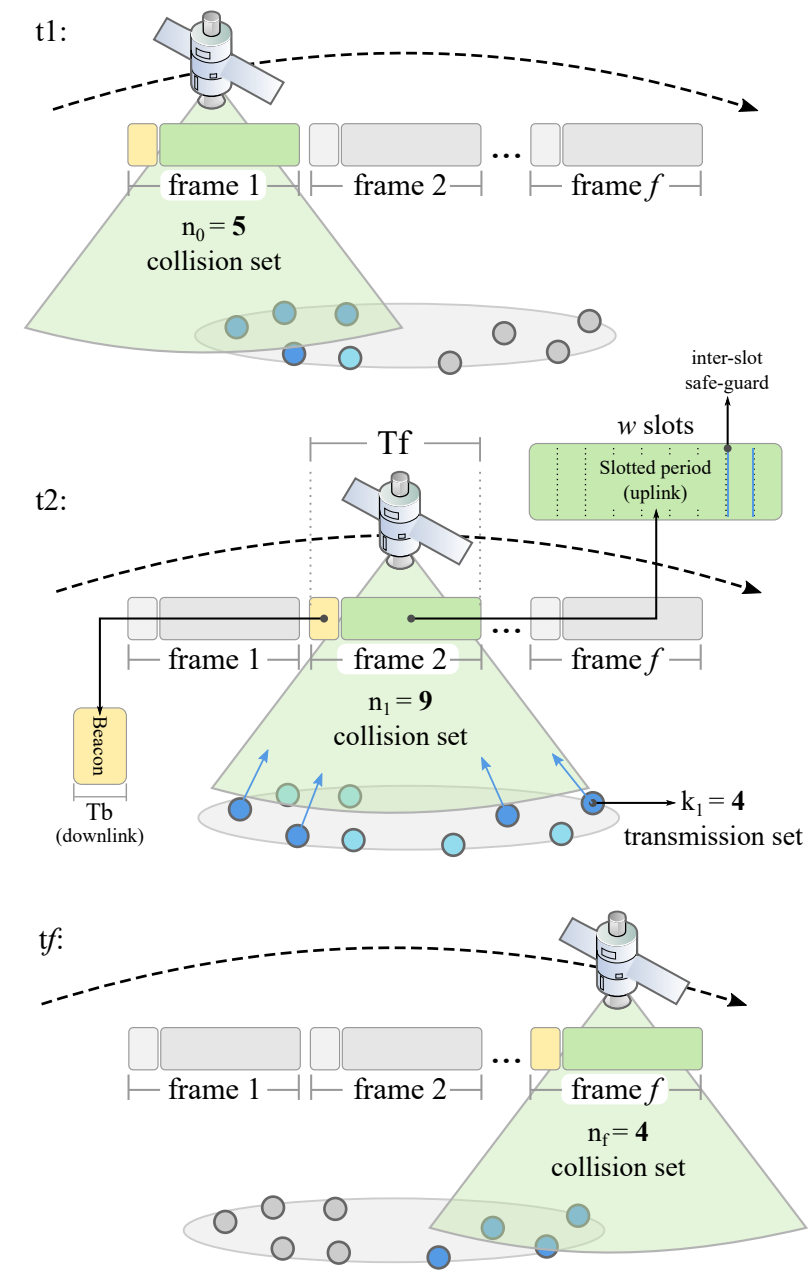

Fig. 1. DtS-IoT framed slotted ALOHA model

transmission could occur immediately after the beacon with at least the most robust modulation and coding scheme available.

This model can be, for instance, materialized by standard LoRaWAN Class-B mode [2], where the beacon is modulated by the most robust spreading factor (SF12), while devices use any SF7-12 for the uplink, depending on the measured channel conditions. As discussed in [9], the absence of a passing-by satellite for a period of no more than 2 hours (i.e., beaconless period) is enough to keep a network-wide synchronization, enabling sparse LoRa-based and power-effective DtS-IoT constellations. In a LoRaWAN-based DtS-IoT, the design of the frame size an $\mathrm{d}$ structure will depend on the downlink duty cycle $\alpha$, and the maximum devices' clock drift $\Delta_{m}$. Assuming a satellite pass time $T_{p}$ over a device with $f$ packets to send over $f$ frames (i.e., 1 packet to 1 frame ratio), the frame duration $T_{f}$ is constrained by

$$
\frac{T_{b}}{\alpha} \leq T_{f} \leq \frac{T_{p}+T_{b}-\Delta_{m}}{f+1} .
$$

Indeed, the frame length has to be longer than $\frac{T_{b}}{\alpha}$, where $T_{b}$ denotes the beacon duration. Also, the frame has to be short enough for a device to transmit $f$ times in $f$ different frames, considering the maximum clock drift between two satellite passes. In compliance with LoRaWAN Class-B, a beacon period $T_{f}$ of 120 seconds is feasible, rendering a duty cycle of nearly $1 \%$ assuming SF12 (airtime 1.32 seconds, at 20 Bytes and coding rate $4 / 5$ ).

In our DtS-IoT model, each frame consists of a fixed number $w$ of slots (echoing a well-studied slotted ALOHA variant for LoRaWAN [14]). Each slot is a time episode in which an IoT device can choose to perform an uplink transmission. It picks one of the slots in the current frame with uniform probability to then schedule a packet in such time period, provided it has successfully decoded the beacon and indeed has data ready for uplink. As a result, packets with airtime up to the slot duration can be sent to the satellite; however, adequate safeguard margins should be considered. For instance, the slant range with a LEO over the horizon can be as large as $2,000 \mathrm{~km}$, which implies a $\sim 7 \mathrm{~ms}$ device-to-gateway propagation delay.

If more than one devices pick the same slot, destructive interference or a collision occurs, rendering all colliding messages unusable (we disregard the so-called capture effect [15]). Thus, slots can be classified as idle (not used), extracted (a single transmission that made it to the gateway on the satellite) or collided (two or more collided transmissions lost) [16]. Since for the satellite, many IoT devices willing to transmit might be in view at the same time (comprising the so-called collision set), the likelihood of collisions in DtS-IoT is typically higher than in traditional less-crowded ground IoT deployments. In particular, if there are more transmitting devices than slots in the frame, collisions are guaranteed, unless some devices were refraining from participating in the frame. Our focus is therefore the investigation of a means to limit the number of contending devices (deriving a transmission set, a subset of the collision set), while considering the LEO dynamics.

a) Collision Set Cardinality Estimation: In order to control the cardinality of the transmission set $k$, the cardinality of the collision set $n$ needs to be estimated upfront. Early DtS-IoT deployments will likely allow operators to exploit $a$ priori knowledge of the position and traffic shape of serviced IoT devices. This information can be uploaded to the satellite in advance so that the number of reachable and contending devices can be determined for each frame. However, more automated and dynamic approaches are also conceivable. For instance, the satellite operator might build in machine learning techniques based on patterns recorded from previous passes over the same region (especially applicable for Earth-repeat orbits [17]). On the other hand, well-studied collision set estimation techniques for dynamic frame-length Aloha protocols [18] can be enhanced. Among these, efficient estimators are available [16] which can be adapted to the time-evolving collision set as observed from the passing-by DtS-IoT satellite. The DtS-IoT collision cardinality learning/estimation is thus possible, but the specific means to achieve them are out of the scope of this paper. We do assume that the satellite can profit from such an estimate to beacon derived relevant information to the IoT devices on ground. 


\section{TRANSMission Probability FunCtion}

Knowing the collision set cardinality does, in principle, allow the construction of an explicit schedule assigning a dedicated slot to each device with data to send. Nevertheless, such a coordination would be prohibitive to compute, to maintain, and costly to roll out (wrt. both bandwidth and power), thereby reducing the DtS-IoT network flexibility and scalability. Instead, relevant information related to the collision set cardinality can be included in the beacon with the aim at yielding better performance than plain slotted Aloha. To this end, we design a Transmission Probability Function, TPF, that maps the number of competing nodes to the probability of indeed contending in the current frame. With the collision set cardinality $n$ and the TPF routine available at the satellite, the resulting transmission probability $p$ can be broadcast on each beacon, giving the satellite some degree of control over the cardinality $k$ of the transmission set as a function of $n$. Then, devices participate in the frame (i.e., select one of its $w$ slots and transmit data on it) with this probability. The remainder of this work formalizes and evaluates the value of using $T P F$ in DtS-IoT networks based on framed slotted Aloha.

Our overall goal is to maximize the expected number of successful messages that can be decoded at the gateway. We introduced $p$ as the transmission probability, $n$ as the number of IoT devices on the collision set, and $k$ as the size of the transmission set. Since $k$ depends on the transmission probability $p$, we assign a random variable $K$ that indicates this size. Furthermore, we denote the number of successfully extracted transmissions as $S$, which is also a random variable. In the following, we will derive an expression for the expected value $E[S]$ in relation to $p$ (or $E[S \mid p]$ ). This will give us an estimator $\mathcal{E}(p)$ of the expected packet extraction performance, depending on how we set the transmit probability.

First, we fix $K=k$, as this case is straightforward to analyze: Let $X_{1}, \ldots, X_{k}$ be random variables, such that $X_{i}=1$ if the $i$-th device has a unique slot, and 0 otherwise. Looking at an individual device, we get

$$
P\left[X_{i}=1\right]=\left(1-\frac{1}{w}\right)^{k-1}=E\left[X_{i}\right],
$$

since all of the $k-1$ other devices need to pick a slot that is different from the one picked by device $i$. As these random variables are 1 with the aforementioned probability and 0 otherwise, this probability is equal to their expected value. From these indicator variables, we can derive the expected value of successes $E[S]$, since the expected value is linear:

$E[S \mid K=k]=E\left[\sum_{i=1}^{k} X_{i}\right]=\sum_{i=1}^{k} E\left[X_{i}\right]=k \cdot\left(1-\frac{1}{w}\right)^{k-1}$

Of course, this holds only in the case where the size of the transmission set is exactly $k$. Since the cases for different $k$ are disjoint, however, we can get the combined value of $E[S]$ by weighting these disjoint cases with their respective probability.

All nodes decide independently whether they should compete, based on $p$. Therefore, $K$ follows a binomial distribution, and we can express the probability for an individual transmission set cardinality $k$ as

$$
P[K=k]=\left(\begin{array}{l}
n \\
k
\end{array}\right) \cdot p^{k} \cdot(1-p)^{n-k},
$$

which we can then apply as described above to find $E[S]$ :

$$
\begin{aligned}
E[S] & =\sum_{k=0}^{n} E[S \mid K=k] \cdot P[K=k] \\
& =\sum_{k=0}^{n}\left(\begin{array}{l}
n \\
k
\end{array}\right) \cdot p^{k} \cdot(1-p)^{n-k} \cdot k \cdot\left(1-\frac{1}{w}\right)^{k-1}
\end{aligned}
$$

This rather complicated expression for the estimator can be simplified as follows (proof included in the appendix):

$$
E[S]=n p\left(1-\frac{p}{w}\right)^{n-1}=: \mathcal{E}(p)
$$

This value only depends on the slot count $w$, the collision set cardinality $n$, and on the transmission probability $p$. The former is known and $n$ can be assumed to be estimated as described above. We can therefore pick $p$ such that the estimator is maximized. To find this maximum, take the derivative of $\mathcal{E}$ by $p$ and look at the roots:

$$
\frac{d \mathcal{E}}{d p}(p)=\frac{n}{w}\left(1-\frac{p}{w}\right)^{n-2} \cdot(w-n p)
$$

Indeed, we find a root at $p=w / n$, which indicates a global maximum: It holds that $\mathcal{E}$ is continuous, $\mathcal{E}(0)=0=\mathcal{E}(w)$, and $\mathcal{E}(p)>0$ for $0<p<w$. Thus, the expected success rate $E[S]$ is maximized if $p=w / n$. Of course, if $w$ is greater than $n$, this maximum is outside the valid range $[0,1]$ for $p$. In these cases, the highest expected number of successes we can achieve is at $p=1$.

The transmit probability $p$ that our TPF should return, given a collision set of $n$ nodes and a frame length of $w$ slots can thus be calculated with the following expression:

$$
\operatorname{TPF}(n)=\underset{p \in(0,1)}{\arg \max } \mathcal{E}(p)=\min \left(1, \frac{w}{n}\right)
$$

\section{A. Wasted Transmissions}

There is a specific phenomenon from the LEO satellite pass dynamics that is not properly represented in our model so far, a time-evolving variant of what is known as the hidden-terminal effect. Consider an IoT device that can see the satellite as it passes over the horizon as illustrated in Fig. 2.

Since the satellite moves along its orbit, it might only stay visible for the device for a shorter time than the frame duration $T_{f}$. If a new frame begins in this situation, the final slots of the frame will occur after the satellite has dropped below the horizon. Assuming that this is not known to the device, it might, according to the presented frame slotted Aloha protocol, decide to transmit without the satellite being at sight. These wasted transmissions would never be observed by the satellite, and consequently account for neither a success nor a collision.

In order to improve the accuracy of the transmission set cardinality estimation in this condition, we study two approaches of concrete practical value, as we discuss in Section IV. 


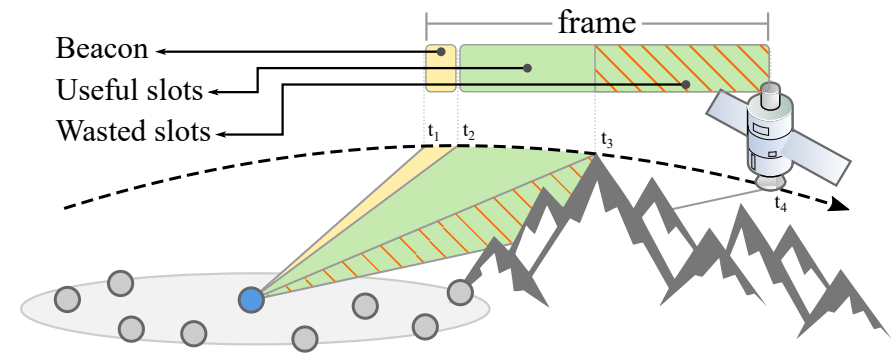

Fig. 2. Useful and wasted slots. Mountains illustrate the horizon of visibility (a.k.a. elevation profile) for the device in blue. Other devices will have a different one, and thus another proportion of useful and wasted slots for the same frame.

a) Estimator Throttling: As we will discuss below, the simulator tool introduced in Section IV enables us to quantify that the relative proportion of wasted transmissions is independent from the transmission probability $p$ (see in Fig. 4 that wasted is a linear expression of $p$ ). Since a fixed percentage of senders waste their messages regardless of the transmit probability, we might as well consider this fixed percentage as non-contending devices in the first place. For instance, at the top of Fig. 4, four sample frames $f=1,2,3,4$ with collision set cardinality $n_{f}$ of 137, 287, 268 and 179 are presented with their resulting wasted transmissions for different $p$ values. Since $W_{c}=38 \%$ of the 287 devices in the second frame waste their transmissions, the throttled estimator $\mathcal{E}^{T}(p)$ would run the original one with collision set cardinality $n=177$, which is $62 \%$ of the total amount of participating devices. In other words, this approach corrects the estimator to maximize the extraction ratio, by ignoring wasted packets. But IoT devices are still performing useless transmissions after the satellite hides behind their individual horizon.

b) Perceptive Devices: To save energy on constrained IoT devices, transmissions when no satellite is at sight should directly be avoided. To this end, we change the devices' behavior to only pick slots where devices can see the satellite. Indeed, we assume some kind of trajectory information is made available to the device via the beacon or other means, an approach already proven practical in Lacuna DtS-IoT satellites [12]. This makes it impossible for wasted transmissions to occur, but it also invalidates the assumption that all slots are picked with equal probability. Therefore, applying $\mathcal{E}(p)$ to perceptive devices might deliver a sub-optimal extraction ratio.

\section{EvaluATiON}

In this section we evaluate the applicability of the presented model and TPF approaches in a realistic DtS-IoT use case.

\section{A. Case Study Setup}

We evaluate a DtS-IoT scenario comprised of 1500 IoT devices deployed uniformly over a circular region on the surface of the planet. We consider a LEO satellite configured with a polar orbit at $600 \mathrm{~km}$ altitude, an inclination of $98^{\circ}$, a right ascension of the ascending node (RAAN) of $20^{\circ}$, and an argument of perigee of $0^{\circ}$ (epoch set to $1 / 1 / 2021$ 00:00:00). The resulting trajectory is computed using the TwoBody propagator included in the Systems Toolkit (STK) from AGI. The resulting dynamics are illustrated in Fig. 3.

We study two coverage configurations regarding the IoT gateway the spacecraft is equipped with. One of them assumes a beamwidth of $90^{\circ}$, the other of $120^{\circ}$. Each antenna is facing the nadir direction, and the $90^{\circ}$ antenna can at any time serve only a subset of the devices in the $120^{\circ}$ configuration.

A simulator that reads the fixed Earth-Center Inertial (ECI) position of the orbital trajectories and IoT devices was developed ${ }^{1}$. The tool is written in RUST, delivering competitive performance and memory safety guarantees while keeping the code clean and readable. For the devices, we included an embedded LUAJIT runtime environment. This enables flexible comparisons across different device behaviors, which we implement in LUA, without recompiling the simulator. We spawn one co-routine per device, and provide an interface to define device's actions, which can dynamically react to other events within the simulation.

We set the frame length to 120 slots of one second each. In the provided scenario, this gives us four useful frames for the $90^{\circ}$ case, and six for the $120^{\circ}$ one. Each of the IoT devices in the evaluated scenario will attempt an uplink on every frame, an aggressive configuration for IoT, convenient to stress the TPF evaluation.

\section{B. Results}

For every possible $p$ in frames in the $90^{\circ}$ and $120^{\circ}$ cases, we study the successful extracted messages and the lost messages, comprising collisions and wasted transmissions (only for nonperceptive devices). These results are collected for simulations with and without perceptive devices (50 seeds for each $p$ for a total of 20k simulations), as well as the expected value of the estimator with and without throttling. Plots are presented in Fig. 4, and described in the caption.

In general, initial and final frames of the two evaluated passes (i.e., frames at the edge of the circular serviced region) present a reduced number of nodes in the collision set $n$ compared to central frames. Indeed, central frames of the $120^{\circ}$ case pose the highest challenge in terms of scalability, with $n_{3}=940$ and $n_{4}=931$ collision set cardinality contending for the 120 slots in the third and fourth frame. Thus, while most congested frames achieve the optimal transmission success with $p<0.2$, initial and final frames are used optimally if $p>0.8$ values are beaconed.

While the estimators theoretically approach the slotted Aloha maximum throughput, simulations not always follow. In particular, estimators for earlier frames at the beginning of the pass provide accurate values, and simulations reach the optimal extraction rates. But as the satellite flies into the service region, more devices start to go out of sight of the gateway and wasted messages become predominant towards the end of the pass. The distribution of devices in these frames

\footnotetext{
${ }^{1}$ https://dgit.cs.uni-saarland.de/kai/dts-iot-sim/
} 

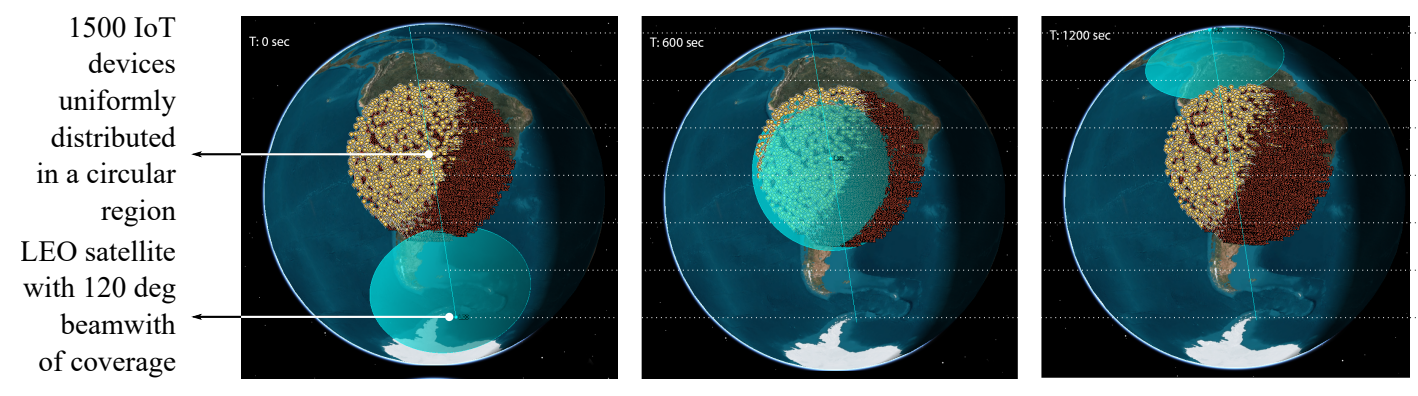

frame 6

frame 5

frame $4 \% 0$

frame $3 \stackrel{\circ \sigma}{\text { वQ }}$

frame 2 \&

frame 1

Fig. 3. Case study DtS-IoT satellite scenario. $90^{\circ}$ and $120^{\circ}$ beamwidth cases are considered.
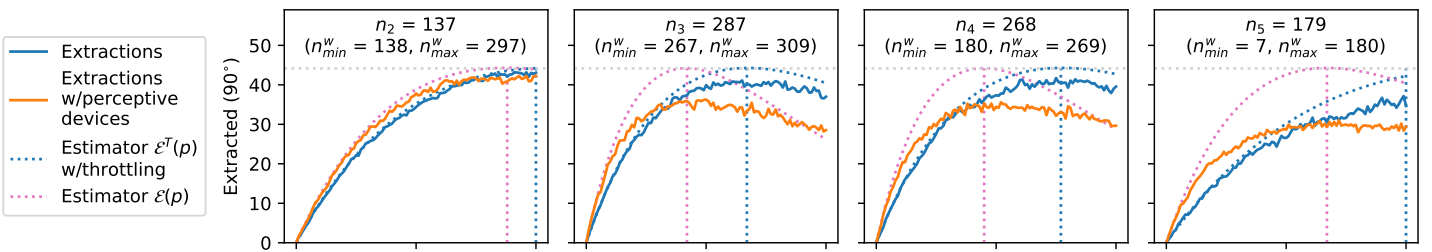

$90^{\circ}$ scenario

(4 frames, on the left)

t.tput: $86 \%$ avg. t.tput percept.: $75 \%$ avg

lost tx: $52 \%$ avg lost tx percept.: $46 \%$ avg
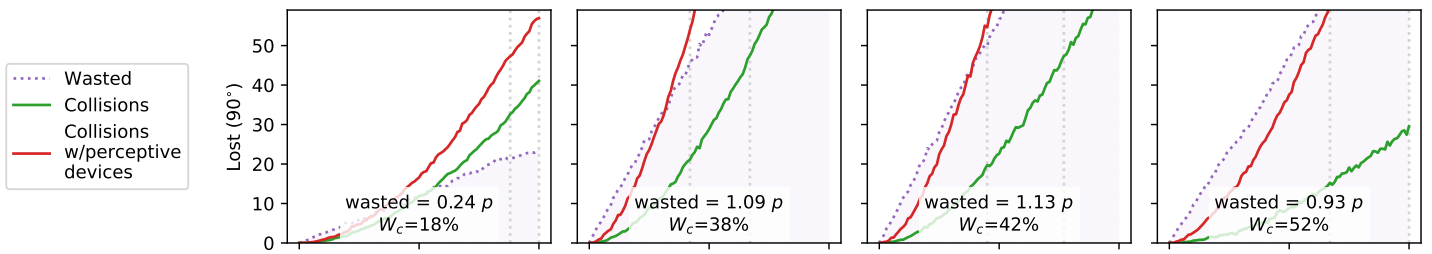

$120^{\circ}$ scenario (6 frames, on the bottom)

t.tput: $89 \%$ avg t.tput percept.: $83 \%$ avg.

lost tx: $25 \%$ avg. lost tx percept.: $23 \%$ avg.
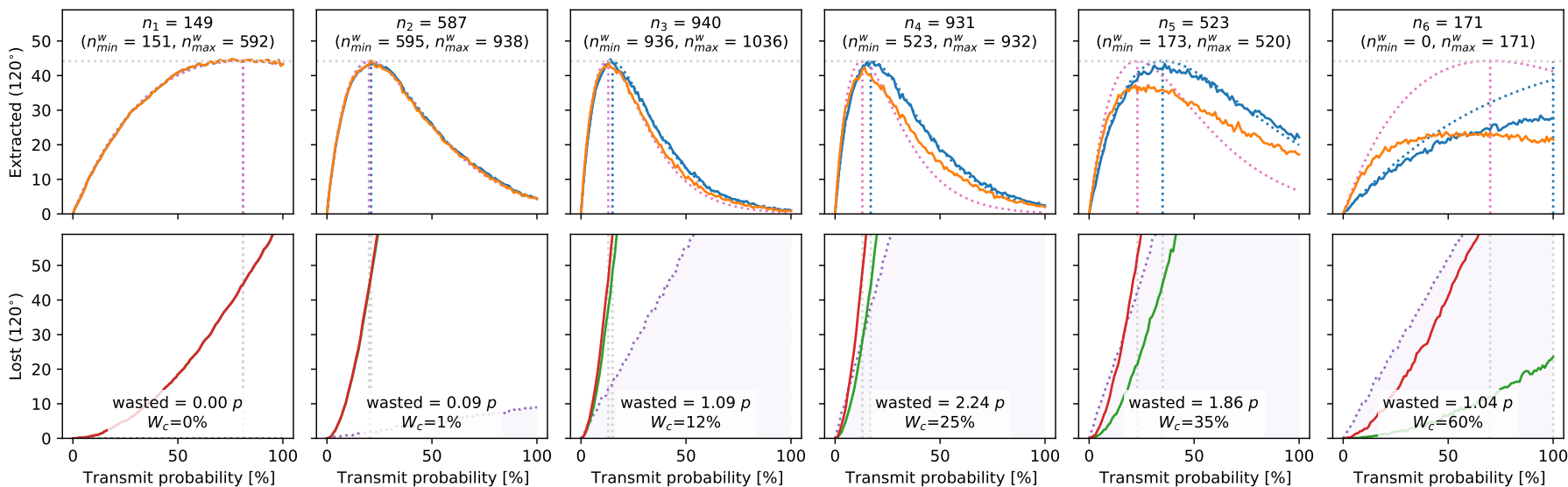

Fig. 4. Evaluation metrics for extraction and lost messages in the $90^{\circ}$ and $120^{\circ}$ coverage scenarios comprising four (top) and six (bottom) useful frames respectively. Plots can be read as a time evolution of the satellite pass from left to right. Simulation and estimator metrics are presented for every possible $p$ for each frame to evaluate the consequences of the $p$ selection. Averaged percentage of the theoretical throughput and percentage of lost transmissions are summarized on the top right. The optimum values of $p$ found by the estimators are marked with a vertical dotted line, and the theoretically achievable throughput upper bound of plain slotted Aloha [13] with 120 slots is marked with a dotted horizontal dotted line (at 44 extracted packets, 36.7\% of the slots). The amount of devices reachable in the beacon of frame $f$ (and considered for the beaconed $p$ at that moment) are noted as $n_{f}$. Also, the min and max nodes reachable in all slots of the frame $\left(n_{\min }\right.$ and $\left.n_{\max }\right)$ are indicated. Note that $n_{\text {max }}$ may be larger than $n_{f}$, since new nodes come into view as the satellite moves in orbit. These nodes did not observe the beacon and are thus not part of the collision set.

is also skewed such that only very few nodes can use the late slots: For instance, in the $90^{\circ}$ case, the number of participants in frame 3 drops from initially 287 (beacon $n_{3}$ ) to 65 , and in frame 5 the count goes down from initially 179 (beacon $n_{5}$ ) to 7 for the very last slot. Note that despite $n_{\min }$ are 267 and 269 for those frames, only 65 and 7 were able to pick up the beacon at the beginning. Indeed, the number of participating devices for which the satellite has not yet passed the horizon can only exhibit a monotonous decrease. This is because devices that are just coming into view did not observe the beacon, and are thus unable to transmit while others are moving out of sight. Since the estimators assume all slots to be equally likely and usable, their accuracy is lowered. This effect is especially pronounced for the later frames where the relative drop is more significant.

a) Estimator Throttling: We still observe the best packet delivery in all frames with the non-perceptive devices, at the transmit probability given by the throttled estimator $(86 \%$ and $89 \%$ of the theoretical throughput). However, this comes with a cost in terms of wasted messages. Since these accumulate with 
collisions to account for the total packet loss, we can conclude that such extraction rates are obtained at the price of energy losses. The throttled approach is however suitable for a multigateway setup on which another satellite has already entered the service region and is in conditions of picking up those otherwise wasted packets. Although multi-gateway approaches are part of the LoRaWAN IoT specification, their study for DtS-IoT based on TPF is left as future work.

b) Perceptive Devices: On the other hand, the perceptive devices completely avoid the waste effect, at the expense of a higher collision rates (at the end of the pass, where more devices will compete for the earlier slots of the last frame) and a slightly sub-optimal extraction ratio (75\% and $83 \%$ ). In both throttled estimator and perceptive devices approaches, the obtained metrics outperform the baseline (i.e., $p=1$ ).

\section{CONCLusion}

A global Internet of Things is possible if Direct-to-Satellite IoT via LEO satellites can be realized. To this end, existing IoT protocols need to be adapted to cope with unprecedented scale due to the large satellite coverage combined with a fastpaced network dynamics imposed by the passing-by gateway in LEO. In this work, we approached the challenge with a framed slotted Aloha model implementable by means of minor adaptations of the LoRaWAN standard. We proposed specific collision cardinality estimators and derived solutions to control the contention of devices under coverage even in demanding LEO dynamics. Simulation results on a realistic case study proved that $87 \%$ of the theoretical throughout can be achieved in practice, although with $38 \%$ of useless transmissions. A perceptive device with enhanced intelligence on the satellite trajectory delivered $79 \%$ of optimality at a lower overhead of $34 \%$ lost packets. Future work includes expanding the estimator to model the waste phenomenon, which could be approached by changing the $X_{i}$ in Equation 2 to not count transmissions that would be wasted. Improvements on the perceptive approach where the slot selection probability might be twisted to favoring late vs. early slots to counter the skew in slot usability are on also on the roadmap.

\section{APPENDIX}

Proof of Equation 3: We can start counting at 1 because the first term of the sum is zero:

$$
\sum_{k=1}^{n}\left(\begin{array}{l}
n \\
k
\end{array}\right) \cdot p^{k} \cdot(1-p)^{n-k} \cdot k \cdot\left(1-\frac{1}{w}\right)^{k-1}
$$

$$
\begin{aligned}
& \text { We apply }\left(\begin{array}{l}
n \\
k
\end{array}\right) \cdot k=\left(\begin{array}{l}
n-1 \\
k-1
\end{array}\right) \cdot n \\
& \quad=n \cdot \sum_{k=1}^{n}\left(\begin{array}{l}
n-1 \\
k-1
\end{array}\right) \cdot p^{k} \cdot(1-p)^{n-k}\left(1-\frac{1}{w}\right)^{k-1}
\end{aligned}
$$

Now we perform an index shift $(j=k-1)$

$$
\begin{aligned}
& =n \cdot \sum_{j=0}^{n-1}\left(\begin{array}{c}
n-1 \\
j
\end{array}\right) \cdot p^{(j+1)} \cdot(1-p)^{n-(j+1)}\left(1-\frac{1}{w}\right)^{j} \\
& =n p \cdot \sum_{j=0}^{n-1}\left(\begin{array}{c}
n-1 \\
j
\end{array}\right)\left(p \cdot\left(1-\frac{1}{w}\right)\right)^{j} \cdot(1-p)^{(n-1)-j}
\end{aligned}
$$

Finally, we apply the binomial theorem

$$
=n p \cdot\left(p \cdot\left(1-\frac{1}{w}\right)+(1-p)\right)^{n-1}=n p\left(1-\frac{p}{w}\right)^{n-1}
$$

\section{ACKNOWLEDGMENT}

This research has received support from the ERC Advanced Grant 695614 (POWVER) and by the DFG Grant 389792660 , as part of TRR 248 (https://perspicuous-computing.science). It has also been supported by the Project STICAMSUD 21STIC-12.

\section{REFERENCES}

[1] A. Osseiran, J. F. Monserrat, and P. Marsch, 5G Mobile and Wireless Communications Technology. Cambridge University Press, 2016.

[2] LoRa Alliance Tech. Committee, LoRaWAN ${ }^{\mathrm{TM}}$ 1.1 Spec., Oct. 2017, v1.1.

[3] G. Boquet, P. Tuset-Peiro, F. Adelantado, T. Watteyne, and X. Vilajosana, "LR-FHSS: Overview and Performance Analysis," 2020. [Online]. Available: https://arxiv.org/abs/2010.00491v3

[4] S. Cluzel, L. Franck, J. Radzik, S. Cazalens, M. Dervin, C. Baudoin, and D. Dragomirescu, "3GPP NB-IOT coverage extension using LEO satellites," IEEE Vehicular Technology Conference, vol. 2018-June, pp. $1-5,2018$.

[5] I. Leyva-Mayorga, B. Soret, M. Röper, D. Wübben, B. Matthiesen, A. Dekorsy, and P. Popovski, "LEO Small-Satellite Constellations for 5G and Beyond-5G Communications," IEEE Access, vol. 8, pp. 184955 $184964,2020$.

[6] R. S. Sinha, Y. Wei, and S.-H. Hwang, "A Survey on LPWA Technology: LoRa and NB-IoT," ICT Express, vol. 3, no. 1, pp. 14 - 21, 2017.

[7] Cisco, "Cisco annual internet report (2018-2023) white paper," 2020.

[8] M. De Sanctis, E. Cianca, G. Araniti, I. Bisio, and R. Prasad, "Satellite Communications Supporting Internet of Remote Things," IEEE Internet of Things Journal, vol. 3, no. 1, pp. 113-123, Feb 2016.

[9] J. A. Fraire, S. Henn, F. Dovis, R. Garello, and G. Taricco, "Sparse satellite constellation design for lora-based direct-to-satellite internet of things," in GLOBECOM 2020. IEEE, 2020, pp. 1-6.

[10] J. A. Fraire, S. Céspedes, and N. Accettura, "Direct-to-satellite iot: A survey of the state of the art and future research perspectives," 62019 , pp. $1-12$.

[11] ITU, "Itu global and regional ict data," 112020.

[12] The Things Network Global Team, LoRa World Record Broken, 2020 (accessed May 28, 2021). [Online]. Available: https://www.thethingsnetwork.org/article/ lorawan-world-record-broken-twice-in-single-experiment-1

[13] L. Kleinrock and S. S. Lam, "Packet switching in a multiaccess broadcast channel: Performance evaluation," IEEE Trans. Commun., vol. 23, no. 4 , pp. 410-423, 1975.

[14] L. Beltramelli, A. Mahmood, P. Osterberg, and M. Gidlund, "Lora beyond aloha: An investigation of alternative random access protocols," IEEE Transactions on Industrial Informatics, 2020.

[15] Č. Stefanović, M. Momoda, and P. Popovski, "Exploiting capture effect in frameless aloha for massive wireless random access," in 2014 IEEE WCNC conference. IEEE, 2014, pp. 1762-1767.

[16] A. Zanella, "Estimating collision set size in framed slotted aloha wireless networks and rfid systems," IEEE Comms. Letters, vol. 16, no. 3, pp. 300-303, 2012.

[17] M. Oliver and G. Eberhard, "Satellite orbits: models, methods and applications," Springer-Verlag Berlin Herdelberg, pp. 1-22, 2000.

[18] F. Schoute, "Dynamic frame length aloha," IEEE Trans. on communications, vol. 31, no. 4, pp. 565-568, 1983. 